\title{
Influencia de los sucesos vitales y el apoyo social en una intervención psicoeducativa para mujeres con depresión
}

Ma Asunción Lara, D ra en C, ${ }^{(1)}$ Claudia N avarro, Lic en Psic, ${ }^{(2)}$ Laura N avarrete, Lic en Psic. ${ }^{(2)}$

\section{Lara MA, Navarro C, Navarrete L. Influencia de los sucesos vitales y el apoyo social en una intervención psicoeducativa para mujeres con depresión. \\ Salud Publica Mex 2004;46:378-387.} El texto completo en inglés de este artículo está disponible en: http://www.insp.mx/salud/index.html

\section{Resumen}

Objetivo. Investigar la influencia del apoyo social y los sucesos vitales so bre los síntomas de depresión: pretratamiento, postratamiento (15-30 días) y seguimiento (cuatro meses), en una intervención psicoeducativa para depresión. Material y métodos. Se seleccionaron 254 mujeres con síntomas de depresión, de entre quienes solicitaron atención para dichos síntomas, en tres centros comunitarios de salud mental y un centro de salud de la Secretaría de Salud, en la Ciudad de México, entre enero de 1998 y diciembre de 2000. La intervención había mostrado previamente su eficacia en reducir los síntomas de depresión. Dichos síntomas se evaluaron con la Escala de D epresión del Centro de Estudios Epidemiológicos (CES-D), y los sucesos vitales y el apoyo social con escalas específicas para estos aspectos. Se realizaron análisis de regresión jerárquica para probar los diversos modelos. Resultados. Modelo 1: efecto de sucesos vitales, apoyo social y variables sociodemográficas (edad, escolaridad, ingreso y ocupación) sobre CES-D pretratamiento. El modelo fue significativo $(p<0.000)$ y las dos primeras variables y edad entraron en el modelo. Modelo 2: efecto de las mismas variables predictoras so bre CES-D postratamiento. El modelo fue significativo $(p<0.001)$; apoyo social y sucesos vitales fueron incluidas en este modelo. Modelo 3: efecto de las mismas variables sobre CES-D en seguimiento. El modelo fue significativo $(p<0.000)$ y las va-

\section{Lara MA, Navarro C, Navarrete L.}

The influence of life events and social support

in a psycho-educational intervention

for women with depression.

Salud Publica Mex 2004;46:378-387.

The English version of this paper

is available at: http://www.insp.mx/salud/index.html

Este proyecto fue parcialmente financiado por el Consejo N acional de Ciencia y Tecnología (CO N ACyT). Proyecto 26026-H.

1) Departamento de Investigaciones Epidemiológicas. Dirección de Investigaciones Epidemiológicas y Psicosociales. Instituto $\mathrm{N}$ acional de Psiquiatría Ramon de la Fuente Muñiz. México, DF, México.

2) Departamento de Investigaciones Epidemiológicas. Instituto N acional de Psiquiatría Ramón de la Fuente Muñiz. México, DF, México.

Fecha de recibido: 19 de agosto de 2003 - Fecha de aprobado: 9 de julio de 2004

Solicitud de sobretiros: D ra. Ma A sunción Lara. Departamento de Investigaciones Epidemiológicas, Dirección de Investigaciones Epidemiológicas. Instituto N acional de Psiquiatría Ramón de la Fuente Muñiz. Calzada México-Xochimilco 101, San Lorenzo Huipulco 14370, México, DF, México. Correo electrónico: laracan@ imp.edu.mx 
riables significativas fueron sucesos vitales y escolaridad. Modelo 4: efecto de apoyo social y sucesos vitales en seguimiento sobre CES-D en seguimiento. El modelo también fue significativo $(p<0.000)$ y ambas variables entraron en el modelo. Análisis adicionales para los modelos 2 y 3 , introduciendo CES-D pretratamiento como variable predictora, mostraron que sólo ésta fue significativa. Conclusiones. Los sucesos vitales y el apoyo social se relacionan con los síntomas de depresión iniciales y también afectan la reducción de dichos síntomas, posteriores a la intervención psicoeducativa. Esto último, en gran medida, se debe a la alta correlación de los sucesos vitales y el apoyo social con la CES-D inicial. Los sucesos y la falta de apoyo en el seguimiento también tienen influencia sobre la CES-D en este periodo. El texto completo en inglés de este artículo está disponible en: http://www.insp.mx/salud/index.html

Palabras clave: depresión; apoyo social; sucesos vitales; mujeres; intervención psicoeducativa; México and life events during follow-up on CES-D during follow-up. The model was also significant $(p<0.000)$ and both variables were included in the model.Additional analyses for models 2 and 3 adding C ES-D pre-treatment as a predictor showed that only the latter was significant. Conclusions. Life events and social support are associated with initial depression symptoms and also predict the reduction of symptoms following the psycho-educational inter vention. This may be due to the high correlation of life events and social support with CES-D pre-treatment. Life events and social support following the intervention also have an effect on CES-D in this same stage.The English version of this paper is available at: http://www.insp.mx/salud/index.html

Key words: depression; social support; life events; women; psycho-educational intervention; Mexico os diversos tipos de adversidad que tienen efecL tos sobre la salud mental se han denominado sucesos vitales (life events) y dificultades -también referidas como crónicas o persistentes-. ${ }^{1}$ Los sucesos vitales son acontecimientos discretos que aparecen en un momento preciso, mientras que las dificultades persistentes son problemas que se prolongan, por al menos cuatro semanas. ${ }^{2}$ Estos sucesos incluyen la vivencia de pérdidas, no sólo personales (la muerte o separación de la pareja), sino también de un papel social (ser corrido del trabajo) o de una idea (descubrir el comportamiento delictivo de un hijo). ${ }^{3, *}$ Los sucesos vitales pueden provenir de diversas áreas de la vida: las relaciones, el hogar, la familia, la salud, los hijos, el matrimonio, las obligaciones sociales, el dinero, el descanso y los amigos, y su poder traumático tienen que ver con su traducción subjetiva.

Se han estudiado la relación entre los sucesos vitales y la ocurrencia de un episodio depresivo, su efecto sobre el resultado de los tratamientos -farmacológicos o psicológicos- y su papel en la gravedad de los síntomas de depresión. Los resultados indican que los sucesos vitales tienen un papel importante en desencadenar un episodio depresivo ${ }^{3,4} \mathrm{y}$, aunque no de manera uniforme, ${ }^{5}$ se considera que éstos disminuyen la posibilidad de mejoría derivada de los tratamientos y aumentan las recaídas. ${ }^{6,7}$ Entre los pocos trabajos con intervenciones psicosociales, ya que la mayoría son de

\footnotetext{
* Se utilizará el término sucesos vitales para referirse, tanto a éstos, como a las dificultades persistentes.
}

tipo farmacológico, Muñoz y Ying, ${ }^{8}$ en quienes se basa nuestro modelo de intervención, encuentran una reducción de los sucesos vitales como resultado de la intervención y una asociación entre esta reducción y la reducción en síntomas de depresión, al año, pero no a los seis meses. También se ha investigado el efecto de los sucesos vitales que se presentan después de los tratamientos en cuanto al efecto que tienen sobre los resultados de los mismos. En este caso los resultados tampoco demuestran de manera unánime que los sucesos vitales tengan un efecto negativo, ${ }^{5}$ no obstante, un estudio controlado y comparativo, entre tratamiento farmacológico y psicológico, reporta una influencia significativa. $^{9}$

Los sucesos vitales también influyen en la severidad de los síntomas de depresión. ${ }^{10-12}$ En este sentido, Brown y Harris ${ }^{2}$ observan que los mismos sucesos que predicen la aparición de un episodio depresivo también predicen la severidad de los síntomas durante dicho episodio.

No se ha logrado establecer si el apoyo social tiene sólo un efecto protector ante las condiciones de estrés, como se ha visto en algunos casos, ${ }^{13}$ pero no en otros, ${ }^{11,14}$ o si la carencia de éste tiene un efecto directo sobre la depresión, como también se ha reportado. ${ }^{15}$ De acuerdo con algunos autores es posible que tenga ambos efectos. ${ }^{16} \mathrm{El}$ apoyo social es un constructo amplio -a veces considerado como un metaconstructo- que, con frecuencia, se define según los propósitos de cada estudio. ${ }^{17}$ Este puede referirse al número o densidad de la red social de apoyo, a la calidad de ésta, a la percepción subjetiva respecto de que ciertas conductas brindan apoyo y al grado de intimidad con la pareja, y 
puede aludir a aspectos emocionales o a instrumentales. ${ }^{18}$ Ejemplo del primero es escuchar con empatía, y del segundo, prestar dinero o compartir tareas.

Entre las diferentes formas de apoyo, se ha investigado el papel que juega contar con alguien en quien confiar. En este sentido, se ha visto que un solo confidente es suficiente para modular los efectos del estrés, lo que no resulta de un amplio espectro de contactos sociales. ${ }^{19}$ En las mujeres, contar con una relación cercana de confianza, protege ante situaciones adversas. ${ }^{15}$ La falta de apoyo social también parece tener un efecto negativo sobre los tratamientos, ${ }^{9,20}$ aunque no todos los estudios han podido demostrar esta relación. ${ }^{21}$ Por último, la carencia de apoyo social también se ha visto que afecta el grado de severidad de los síntomas de depresión. ${ }^{11}$

En cuanto a las variables sociodemográficas, se observa que en las mujeres hay un incremento paulatino en la prevalencia de depresión de la infancia a la edad adulta. ${ }^{22}$ En nuestro país, estudios transversales muestran mayor frecuencia de trastornos depresivos en el grupo de edad de 30 a 59 años. ${ }^{23}$ Respecto al estatus socioeconómico, los mismos autores reportan mayor prevalencia de depresión en personas con menores ingresos, y otros estudios señalan una mayor frecuencia en mujeres con menor escolaridad. . $^{4, *}$ Por otra parte, menor depresión se encuentra en mujeres que trabajan fuera del hogar. ${ }^{25}$

La intervención para mujeres con depresión -a la que se refiere este trabajo- es de tipo psicoeducativo, esto es, proporciona información respecto de qué es la depresión y sobre los principales factores de riesgo de la misma y aplica técnicas psicológicas para reducir los síntomas de depresión. Está estructurada alrededor de un material educativo ${ }^{26}$ y su efectividad ha sido reportada con anterioridad. ${ }^{27-29}$ Dichos trabajos han mostrado reducción significativa de los síntomas de depresión. ${ }^{27}$ Inicialmente, se diseñó como una intervención preventiva para mujeres en riesgo de depresión, pero debido a las características de la población a la que se quería beneficiar, se amplió a una intervención temprana, esto es, para mujeres con un diagnóstico de depresión moderada, como se describe más adelante. ${ }^{27,30}$

\footnotetext{
* Aguilar-Morales LV, Salgado de Snyder VN, Cruz-Valdez A, Zamora-Muñoz JS, Lazcano-Ponce EC. Factores asociados a sintomatología depresiva en mujeres de Morelos, México. Un estudio con base poblacional. Cuernavaca, Morelos, México: Dirección de Epidemiología, Centro de Investigación en Salud Poblacional, Instituto Nacional de Salud Pública; 2002. Documento no publicado.
}

El objetivo del presente trabajo fue analizar la influencia de los sucesos vitales y el apoyo social, controlando las variables sociodemográficas antes mencionadas, sobre los síntomas de depresión iniciales y posteriores a la intervención. Un objetivo secundario fue conocer cuáles son los sucesos vitales más frecuentes y el tipo de apoyo social con que cuenta esta población. El trabajo pretende contribuir a la muy escasa literatura en esta área en nuestro país y sugerir elementos para el diseño de intervenciones más específicas y pertinentes para la depresión femenina.

\section{Material y métodos}

Muestra. Se propuso contar con una muestra de 120 mujeres al final de la intervención, para lo cual, en un inicio, se definió seleccionar, de conveniencia, al doble de mujeres, ya que en estos estudios se ha reportado una pérdida elevada de sujetos. ${ }^{31}$ La muestra se tomó de la población de mujeres que habitualmente asiste a tres centros comunitarios de salud mental y un centro de salud de la Secretaría de Salud, en la Ciudad de México, entre enero de 1998 y diciembre de 2000. La muestra se conformó mediante un proceso de promoción, tanto en la comunidad como entre las pacientes de los servicios, que consistió en invitar a recibir ayuda -por medio de carteles, folletos y contacto personal- a quienes consideraban que tenían síntomas de depresión. A las interesadas se les citó para la evaluación pretratamiento, $\mathrm{y}$ a todas las que cumplieron con los criterios del estudio se les asignó a un grupo. La asignación no fue aleatoria y lo que se asignó fue una intervención determinada a un servicio determinado, según las características de los mismos.

Los criterios globales de elegibilidad de la muestra fueron: a) edad entre 20 y 45 años, b) escolaridad mínima para la lectura del material educativo, y c) sintomatología depresiva moderada (de 25 a 30) o severa $(<31)$ en la Escala de Depresión del Centro de Estudios Epidemiológicos (CES-D). ${ }^{32}$ Los criterios de exclusión fueron: a) presentar un diagnóstico de daño orgánico, psicosis o trastorno bipolar (entrevista clínica estructurada para el DSM-III-R -SCID-, por sus siglas en inglés; ${ }^{33}$ b) estar bajo tratamiento psicológico o farmacológico en el momento del estudio; c) enfermedad terminal; d) adicción a sustancias; e) ideación suicida; f) mostrar evidencia de incapacidad severa debida a su estado de depresión, y g) estar embarazada.

Diseño. El estudio fue comparativo entre una intervención grupal (IG) y una individual mínima (IIM), con mediciones pre, postratamiento (a los 15 días en la IG y al mes en la IIM) y seguimiento a los cuatro meses. Para los fines de este trabajo se tomó a la muestra com- 
pleta para los análisis, debido a que no se encontraron diferencias entre ambas modalidades. ${ }^{27}$

Características de la intervención. La intervención grupal fue conducida por una facilitadora durante seis sesiones semanales, de dos horas cada una, mientras que en la intervención individual se proporcionó orientación, de 20 minutos a una hora, sobre cómo utilizar el material educativo, que se entregó a la participante. Una descripción más amplia sobre las intervenciones puede encontrarse en otros trabajos. ${ }^{27-29}$

Instrumentos. El equipo de investigación fue capacitado previamente para aplicar, a través de entrevistas, los instrumentos de medición de pre, postratamiento y seguimiento. Estos instrumentos se integran por las secciones siguientes:

Variables sociodemográficas. En esta sección se evaluaron: a) la edad (años cumplidos); b) nivel de escolaridad: primaria, secundaria, preparatoria o equivalente, preparación profesional o técnica; c) ingreso (total de ingresos en la familia); d) estado civil: casada o en unión libre, separada, divorciada o viuda, soltera, y e) ocupación: trabajo remunerado, ama de casa.

Sintomas de depresión. Se evaluó con la CES-D. ${ }^{32}$ Es una escala de autorreporte de 20 reactivos que investiga la frecuencia con la que el síntoma se ha presentado en el último mes. Se utilizó la versión de Medina Mora y colaboradores $^{34}$ de tres opciones de respuesta en las que se toma la suma del valor numérico para su calificación ( $1=$ nunca o casi nunca, $2=$ ocasionalmente, $3=$ casi siempre o todo el tiempo).

Sucesos vitales. Se utilizó una escala de 23 reactivos sobre sucesos vitales y dificultades persistentes, ocurridos durante los seis meses previos a la entrevista, en las áreas de familia, pareja, salud, economía e hijos, entre otras. Se evaluó su presencia o ausencia, y con cuatro opciones de respuesta, el grado de tensión asociado a cada suceso $(0=$ no ocurrió el suceso u ocurrió y no produjo estrés; $1=$ ocurrió y produjo muy poco estrés; $2=$ ocurrió y produjo estrés moderado; $3=$ ocurrió y produjo mucho estrés). La escala es una adaptación de Holmes y Rahe ${ }^{35}$ e incluye elementos de otras escalas. ${ }^{2,36}$ No cubre todos los posibles sucesos, como tampoco lo hacen versiones más amplias. ${ }^{2}$

Apoyo social. Se evaluó de manera subjetiva a partir de cuatro reactivos que indagan si la persona cuenta: a) con un confidente, b) con ayuda en caso de necesidad económica, c) con alguien que proporcione información, y d) si se siente cercana a su pareja. Las respuestas son dicotómicas y aunque la escala es muy sencilla, formas similares han sido utilizadas en diversos estudios. ${ }^{11,37,38}$ La calificación se obtiene sumando los apoyos con los que cuenta la persona.
Aspectos éticos. Se obtuvo consentimiento informado de acuerdo con lo que establecen los "Principios éticos para investigación médica en seres humanos", de la Declaración de Helsinki.

Plan de análisis. Se estimaron las frecuencias, porcentajes, medias y desviaciones estándar para las variables sociodemográficas, sucesos vitales y apoyo social, así como diferencias de medias y correlaciones entre algunos de estos aspectos y los síntomas de depresión. Para evaluar las hipótesis sobre la relación entre las variables del estudio y los síntomas de depresión se llevaron a cabo análisis de regresión jerárquica.

\section{Resultados}

Se aplicó la entrevista pretratamiento a 254 mujeres, de las cuales 154 concluyeron con la intervención y fueron evaluadas en postratamiento; 135 de éstas también fueron evaluadas en el seguimiento.*

Características sociodemográficas. Como puede observarse en el cuadro I, la media en síntomas de depresión (CES-D) fue de 40.79 (DE= 8.70; rango: 20-60), siendo la media poblacional de $25 .{ }^{34}$ La media de edad fue de 35.3 años (DE= 6.57) y de ingreso, $\$ 3546.39$ mensuales (que corresponde al tercer cuartil de ingreso de los hogares, o sea habría dos segmentos poblacionales de menores ingresos). ${ }^{39}$ En escolaridad, la mayoría tenía preparatoria o equivalente $(38.7 \%)$ y la minoría sólo primaria $(16.2 \%) ; 22.9 \%$ secundaria y $22.2 \%$ estudios profesionales o técnicos. Respecto a la ocupación, más de la mitad realizaba alguna actividad económica: empleada asalariada $(36.6 \%)$, vendedora o autoempleada no asalariada $(25.2 \%)$, servicio doméstico $(13.4 \%)$, maestra primaria $(8.0 \%)$ y otros $(17.8 \%)$; mientras que el estado civil correspondió a: $65.7 \%$ unida a una pareja, $19.7 \%$ separada, viuda, o divorciada, y $14.6 \%$ soltera.

En el cuadro I también se muestran los resultados de la relación entre las variables sociodemográficas y los síntomas de depresión (anteriores a la intervención) (CES-D). Las correlaciones (Pearson) para edad e ingreso mostraron que sólo la segunda tuvo una correlación significativa $(p<0.000)$ : a menor ingreso más síntomas de depresión. En el análisis de varianza para síntomas de depresión, según nivel de escolaridad se

\footnotetext{
* De la evaluación inicial a la primera sesión se perdió 25\% de la muestra, y entre la primera y segunda sesión, 19\%; $6 \%$ durante el resto de la intervención y $14.8 \%$ en las evaluaciones de postratamiento y seguimiento. Al analizar los resultados se pudo demostrar que quienes desertaron no tuvieron más síntomas de depresión que quienes permanecieron. ${ }^{27}$
} 


\section{Cuadro I \\ Características sociodemográficas y Síntomas de depresión. Ciudad de México, 1998-2000}

\begin{tabular}{|c|c|c|c|c|c|}
\hline & & & & CE. & \\
\hline & & Media & $\mathrm{DE}$ & $r$ & $p$ \\
\hline Depresión (CES- & & 40.79 & 8.67 & & \\
\hline Edad & & 35.32 & 6.57 & -0.103 & 0.102 \\
\hline Ingreso & & 3546.39 & 2285.26 & -0.262 & 0.000 \\
\hline & & & CES- & & \\
\hline & frec $(\%)$ & Media & $\mathrm{DE}$ & ANOVA & \\
\hline Escolaridad & & & & $f$ & $p$ \\
\hline Primaria & $41(16.2)$ & 42.12 & 7.41 & & \\
\hline Secundaria & $58(22.9)$ & 43.13 & 7.20 & 3.45 & 0.01 \\
\hline Preparatoria & $98(38.7)$ & 39.97 & 9.10 & & \\
\hline Profesional & $56(22.2)$ & & & & \\
\hline Total & $253(100)$ & 38.47 & 9.38 & & \\
\hline & & & CES- & & \\
\hline & frec $(\%)$ & Media & $\mathrm{DE}$ & $t$ & $p$ \\
\hline 0 cupación & & & & & \\
\hline Sí & $164(64.6)$ & 39.98 & 9.23 & -2.02 & 0.04 \\
\hline No & $90(35.4)$ & 42.28 & 7.36 & & \\
\hline Total & $254(100)$ & & & & \\
\hline $\begin{array}{l}n=254 \\
\text { CES-D: Escala d }\end{array}$ & presión de & antro d & tro & mint & \\
\hline
\end{tabular}

observó que mayor depresión se asoció con menor escolaridad $(p<0.01)$. Finalmente, las diferencias de medias entre mujeres que trabajan y no, resultó también significativa $(p<0.04)$ : mayor depresión se observó entre quienes no trabajan.

Sucesos vitales. La media en la escala de pretratamiento fue de 12.23 (DE 7.19; rango: 0-36). En el cuadro II se presentan los porcentajes de mucho estrés o malestar (con calificación de 3) de cada reactivo. Entre éstos, los más significativos son: dificultades para educar a los hijos, cambios en la manera de comportarse de un familiar (alguien que era atento deja de serlo, se vuelve más enojón o algún miembro de la familia comienza a beber), problemas económicos serios, enfermedad de un familiar, amenazas de la pareja, dificultades con parientes (que no sean hijos y pareja), cambios en la frecuencia con la que ve a familiares (ven poco a quienes desearían ver o más frecuentemente a quienes no) y consumo de alcohol o drogas de la pareja.

$\mathrm{Al}$ analizar la correlación (Spearman) entre cada suceso vital (tomando en cuenta los cuatro valores con los que se califica cada ítem) y la CES-D se vio que sólo cuatro de ellos tuvieron una correlación significa-

\section{Cuadro II \\ SUCESOS VITALES QUE PRODUCEN MAYOR TENSIÓN. Ciudad de México, 1998-2000}

\begin{tabular}{lc} 
& $f(\%)^{*}$ \\
A bandono o divorcio & $13(5.1)$ \\
\hline Consumo de alcohol o drogas de la pareja & $34(13.4)$ \\
\hline Amenazas de la pareja & $61(24.0)$ \\
\hline Golpes de la pareja & $13(5.1)$ \\
\hline Violencia sexual de la pareja & $15(5.9)$ \\
\hline Jubilación o pérdida de trabajo & $30(11.8)$ \\
\hline Cambio de trabajo & $15(5.9)$ \\
\hline Problemas económicos serios & $97(38.2)$ \\
\hline Enfermedad de un familiar & $73(28.7)$ \\
\hline Cambios en frecuencia de ver familiares & $52(20.5)$ \\
\hline Muerte de un amigo & $8(3.1)$ \\
\hline Separación familiar & $26(10.2)$ \\
\hline Cambios en la conducta de un familiar & $102(40.2)$ \\
\hline Muerte de la pareja o familiar cercano & $19(7.5)$ \\
\hline Aborto & $1(0.4)$ \\
\hline Problemas legales & $23(9.1)$ \\
\hline Dificultades con parientes & $54(21.3)$ \\
\hline Enfermedad personal & $23(9.1)$ \\
\hline Violencia sexual, no de la pareja & - \\
\hline Cambio de domicilio & $14(5.5)$ \\
\hline A buso físico, no de la pareja & $9(3.5)$ \\
\hline Dificultades para educar a los hijos & $158(62.2)$ \\
\hline Consumo de alcohol o drogas en hijos & \\
\hline & \\
\hline
\end{tabular}

tiva: dificultades para educar a los hijos $(r=0.160 ; p<0.02)$, cambios en la frecuencia con que ve a un familiar $(r=0.242$; $p<0.008)$, enfermedad de un familiar $(r=0.228 ; p<0.01)$ y enfermedad personal $(r=0.431 ; p<0.008)$. También se valoró la relación entre toda la escala y los síntomas de depresión (Pearson) y se observó una correlación significativa $(r=467 ; p<0.000)$.

Apoyo social. Como puede verse en el cuadro III, el apoyo social con el que la mayoría cuenta es para resolver problemas económicos (67.7\%); seguido de apoyo para obtener información $(57.9 \%$ ) y, en tercer lugar, el apoyo de un/una confidente ( $48 \%$ ). El menos frecuente es el de cercanía con la pareja (41.9\%), en el cual se consideró únicamente a quienes la tenían. La media del número de apoyos al inicio fue $2.01(\mathrm{DE}=1.16$, rango: 0-4); $10 \%$ mencionó no contar con ninguno.

Se evaluó si había diferencias en síntomas de depresión entre quienes contaban y no con cada uno de 


\section{Cuadro III \\ Apoyo SOCIAL Y Síntomas DE DEPREsión. CiUdAd de México, 1998-2000}

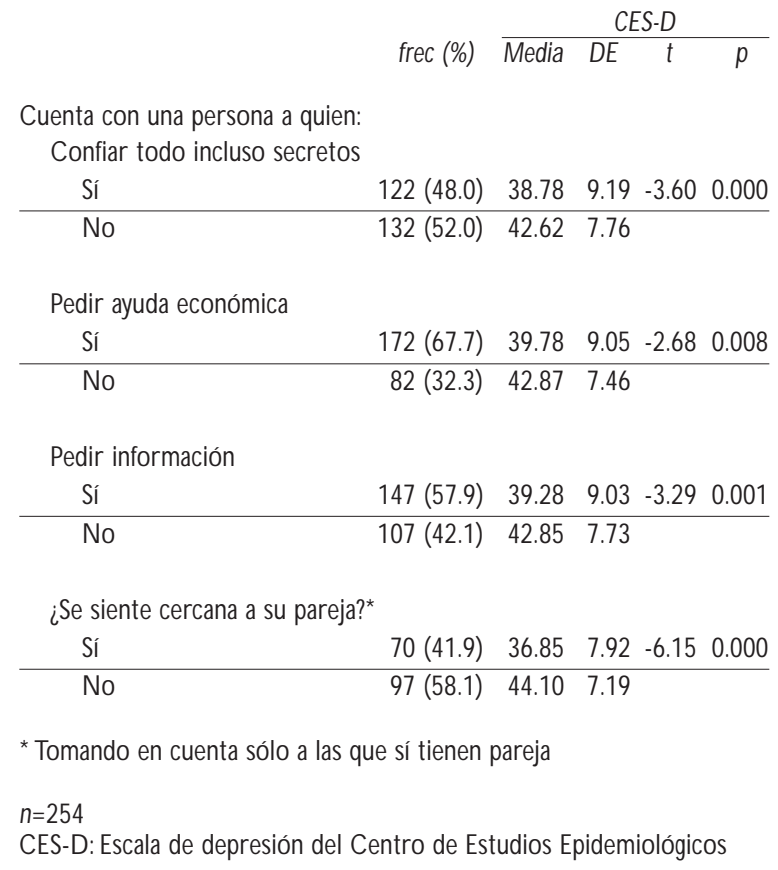

los apoyos, por medio de pruebas ' $t$ '. Se encontró que, en todos los casos, no disponer del apoyo mencionado se asoció, de manera significativa, con más síntomas de depresión (cuadro III). Se llevó a cabo un análisis adicional para comparar si había diferencias en síntomas de depresión entre quienes tenían y no pareja, para evaluar si la falta de pareja podía representar en sí carencia de apoyo, y por lo tanto, mayor depresión. A este respecto no se encontraron diferencias significativas entre quienes tenían y no pareja $(t=0.703 ; p<0.48)$. Por otra parte, se exploró la relación entre la media de la escala con la CES-D observándose una correlación (Pearson) significativa entre ambas $(r=-0.358 ; p<0.000)$, esto es, a menor número de apoyos sociales se incrementó la severidad en los síntomas de depresión.

Relación entre variables y sintomas de depresión. Para evaluar cada uno de los modelos se llevaron a cabo análisis de regresión jerárquica en los que se analizaron las variables predictoras sobre los síntomas de depresión. Las variables sociodemográficas incluidas en todos los modelos fueron: edad, escolaridad, ingreso y ocupación (cuadro IV). En cada modelo se indica el tamaño de la muestra de cada análisis.

Modelo 1. Evaluó el efecto de los sucesos vitales y el apoyo social preintervención sobre la CES-D prein- tervención, controlando por las variables sociodemográficas. Se observó que el modelo fue significativo $(p<0.000)$ y explicó $29 \%$ de la varianza (cuadro IV). Las variables significativas fueron edad, sucesos vitales y apoyo social. Menor edad, mayor número de sucesos y menos apoyo predijeron más síntomas de depresión. Se realizaron análisis adicionales para entender la influencia de la edad, ya que en el análisis bivariado, arriba reportado, la edad no se relacionó con la CESD. Se encontró que, aunque las de menos edad (>35) presentaban más sucesos vitales (13.09 vs 11.93) que las de mayor edad $(<36)(t=1.24 ; p>0.05)$, también reportaron mayor número de apoyos sociales (2.06 vs $1.97 ; t=0.610 ; p>0.05)$, pero las diferencias no fueron significativas. Se investigó entonces la influencia de la edad de los hijos, desde la hipótesis de que las más jóvenes tendrían hijos más pequeños, lo cual podría relacionarse con mayor depresión. Los resultados, sin embargo, mostraron que la mayor depresión se asoció con quienes tenían hijos de entre 12 y 18 años de edad ( $F=5,63, p<0.01)$, que, en su mayoría, fueron las mujeres de mayor edad.

Modelo 2a. Evaluó el efecto de las mismas variables predictoras del modelo 1 , pero sobre el CES-D postratamiento. Los resultados mostraron que el modelo fue significativo $(p<0.001)$ aunque sólo explicó $10 \%$ de la varianza. Las únicas variables significativas fueron sucesos vitales y apoyo social.

Modelo 2b. En este modelo, similar al anterior, se incluyeron como variables predictoras, además de las del modelo 2a (sucesos vitales, apoyo social y sociodemográficas), la CES-D preintervención, sobre CES-D postratamiento. Esto se hizo en vista de que, como se observó en el análisis bivariado y en el modelo 1, los sucesos vitales y el apoyo social tuvieron una alta correlación con la CES-D, con el fin de evaluar si la influencia de dichas variables sobre la CES-D postratamiento era directa o podía estar mediada por su relación con la CES-D inicial. El modelo también fue significativo $(p<0.000)$ y explicó $18 \%$ de la varianza. Como puede verse en el cuadro IV, sólo la CES-D fue significativa: mayor número de síntomas pretratamiento predijo mayor número de síntomas en la CES-D postratamiento.

Modelo 3a. Evaluó el efecto de las mismas variables predictoras de los modelos anteriores sobre la CESD en seguimiento. El modelo fue significativo $(p<0.000)$ y explicó $13 \%$ de la varianza. En este análisis resultaron significativas las variables escolaridad y sucesos vitales: menor escolaridad y mayor número de sucesos predijeron mayor número de síntomas en el seguimiento. Para entender el papel de la escolaridad, se analizaron las diferencias entre las de menor escolaridad (primaria y secundaria) y las de mayor (prepara- 


\section{Cuadro IV \\ ANÁLISIS DE REGRESIÓN LINEAL JERÁRQUICO DE CARACTERÍSTICAS SOCIODEMOGRÁFICAS, SUCESOS VITALES y apoyo social sobre síntomas de depresión. CiUdad de MÉXICO, 1998-2000}

\begin{tabular}{|c|c|c|c|c|c|c|}
\hline Modelo y variable & Beta & DE & $r^{2}$ & $f$ & $\mathrm{t}$ & $p$ \\
\hline \multicolumn{7}{|l|}{ Modelo 1: $(n=254)$} \\
\hline Edad, escolaridad, ingreso, ocupación, SV y AS pre sobre CES-D pre & & 0.29 & 29.51 & 0.000 & & \\
\hline Edad & -0.13 & 0.07 & & & -2.25 & 0.025 \\
\hline SV & 0.41 & 0.07 & & & 6.99 & 0.000 \\
\hline AS & -0.24 & 0.43 & & & -4.22 & 0.000 \\
\hline \multicolumn{7}{|l|}{ Modelo 2a: $(n=154)$} \\
\hline Edad, escolaridad, ingreso, ocupación, SV y AS pre sobre CES-D pos & & & 0.10 & 7.43 & & 0.001 \\
\hline SV & 0.17 & 0.12 & & & 1.99 & 0.048 \\
\hline AS & -0.23 & 0.66 & & & -2.69 & 0.008 \\
\hline
\end{tabular}

\begin{tabular}{|c|c|c|c|c|c|c|}
\hline \multicolumn{7}{|l|}{ Modelo 2b: $(n=154)$} \\
\hline Edad, escolaridad, ingreso, ocupación, SV,AS y CES-D pre sobre CES-D pos & & & 0.18 & 28.56 & & 0.000 \\
\hline CES-D pre & 0.42 & 0.09 & & & 5.34 & 0.000 \\
\hline \multicolumn{7}{|l|}{ Modelo 3a: $(n=135)$} \\
\hline Edad, escolaridad, ingreso, ocupación, SV y AS pre sobre CES-D seguim & & & 0.13 & 8.39 & & 0.000 \\
\hline Escolaridad & -0.24 & 0.83 & & & -2.69 & 0.008 \\
\hline SV & 0.22 & 0.13 & & & 2.48 & 0.014 \\
\hline
\end{tabular}

Modelo 3b: $(n=135)$

Edad, escolaridad, ingreso, ocupación, SV,AS y CES-D pre sobre CES-D seguim

\begin{tabular}{|c|c|c|c|}
\hline Escolaridad & -0.21 & 0.81 & -2.39 \\
\hline CES-D pre & 0.32 & 0.10 & 3.74 \\
\hline
\end{tabular}

Modelo 4: $(n=135)$

\begin{tabular}{lrrrr} 
SV y AS seguim sobre CES-D seguim & & 0.42 & 32.05 & 0.000 \\
\hline SV & 0.55 & 0.10 & 6.36 & 0.000 \\
\hline AS & -0.21 & 0.69 & -2.43 & 0.017
\end{tabular}

SV = sucesos vitales

toria o más) en cuanto a sucesos vitales y apoyo social. Se encontró que las primeras reportaron más sucesos vitales $(t=2.56 ; p<0.03)$ y menor apoyo social $(t=-3.53$; $p<0.000$ ).

Modelo 3b. Este fue similar al anterior pero en éste se incluyó, junto con las variables predictoras, el CESD pretratamiento, sobre la CES-D en seguimiento. El modelo también fue significativo $(p<0.000)$ y explicó $18 \%$ de la varianza. Aquí, las variables significativas fueron la CES-D pretratamiento y escolaridad.

Modelo 4. Evaluó el efecto de las variables, sucesos vitales y apoyo social durante el seguimiento sobre la CES-D, en el seguimiento. El modelo fue significativo $(p<0.000)$ y explicó $42 \%$ de la varianza, resultando significativas ambas variables.

\section{Discusión}

El objetivo del trabajo fue investigar la influencia del apoyo social y los sucesos vitales sobre los síntomas de depresión anteriores y subsecuentes al tratamiento, controlando por las variables sociodemográficas: edad, escolaridad, ocupación e ingreso, en mujeres que participan en una intervención psicoeducativa, así como conocer cuáles son los sucesos vitales más frecuentes y el tipo de apoyo social con que cuenta esta población. El estudio se realizó en la Ciudad de México en centros comunitarios de salud mental y en un centro de salud de la Secretaría de Salud.

Las participantes tuvieron una edad promedio de 35 años, con escolaridad variada: $16 \%$ de primaria, $23 \%$ 
de secundaria, 39\% de preparatoria o equivalente y $22 \%$ profesional o técnico. Más de la mitad trabaja fuera del hogar, aunque no todas de tiempo completo; el ingreso familiar es de alrededor de 3500 pesos al mes, lo cual indica que pertenecen a sectores medio y medio bajo, de acuerdo con la Encuesta Nacional de Salud $2000,{ }^{39}$ lo que no les asegura una vida sin muchas carencias, debido a las condiciones económicas del país. Se observó que las mujeres con menores ingresos, menor escolaridad y que no trabajan tuvieron mayor número de síntomas de depresión, datos que son consistentes con los reportados en la literatura. $3,23-25,40, *$ La edad, por otro lado, no se relacionó con los síntomas de depresión.

Se observó una media de 12 en la escala de sucesos vitales, cifra que combina número y severidad de los mismos. Este es un valor elevado, ya que por sí mismo un solo acontecimiento muy estresante (con valor de 3) puede desencadenar depresión o síntomas considerables. Los sucesos vitales más frecuentes evaluados como más estresantes (con valor de 3) fueron: dificultades para educar a los hijos, cambios en la manera de comportarse de un familiar, problemas económicos serios, enfermedad de un familiar, amenazas de la pareja, dificultades con parientes, cambios en la frecuencia con que ven a familiares y consumo de alcohol o drogas de la pareja. $\mathrm{Al}$ analizar cuáles de ellos se relacionaban con los síntomas de depresión, se encontró que fueron significativos: dificultades para educar a los hijos, cambios en la frecuencia con que ve a un familiar, enfermedad de un familiar y enfermedad personal. En un estudio similar en Suramérica ${ }^{41,42}$ también se observó que los problemas económicos serios, la enfermedad de un familiar y las dificultades con parientes, se relacionaron con síntomas psiquiátricos; estos sucesos están ligados a las características sociales y a las condiciones económicas de la región. Las dificultades que enfrentan las mujeres para educar a los hijos y las derivadas del abuso de sustancias por parte de la pareja y de la violencia de ésta, también se ha encontrado en otros trabajos en nuestro país. ${ }^{43-45}$ Como se esperaba, con base en la literatura ${ }^{10-12}$ la puntuación en la escala de sucesos vitales se relacionó con la severidad de los síntomas de depresión.

\footnotetext{
* Aguilar-Morales LV, Salgado de Snyder VN, Cruz-Valdez A, Zamora-Muñoz JS, Lazcano-Ponce EC. Factores asociados a sintomatología depresiva en mujeres de Morelos, México. Un estudio con base poblacional. Cuernavaca, Morelos, México: Dirección de Epidemiología, Centro de Investigación en Salud Poblacional, Instituto Nacional de Salud Pública; 2002. Documento no publicado.
}

En cuanto al apoyo social que reciben, se vio que cuentan, en promedio, con dos de cuatro de ellos, siendo el menos frecuente el que proviene de la cercanía con la pareja, y $10 \%$ mencionó no contar con ninguno. La carencia de un confidente, de una persona que ayude en caso de problemas económicos o que pueda proveer de información y la falta de cercanía de la pareja se asociaron con mayor número de síntomas de depresión. De la misma manera y conforme se esperaba, ${ }^{11}$ la suma de los apoyos sociales se relacionó con mayor sintomatología. Otros estudios en México también han notificado relación entre la falta de apoyo social y el malestar emocional, aunque utilizando otro tipo de mediciones de apoyo. ${ }^{10}$

En cuanto a la influencia de los sucesos vitales y el apoyo social sobre los síntomas de depresión, controlando por las variables sociodemográficas (modelo 1) se encontró, de nuevo, que ambas variables fueron significativas y también lo fue la edad. Esta última no pudo explicarse por diferencias en sucesos vitales ni apoyo social, ni con base en la edad de los hijos, por lo que se requerirá de profundización en este aspecto en estudios subsecuentes.

Respecto a la influencia de los acontecimientos vitales y el apoyo social, sobre los síntomas de depresión en el postratamiento y en el seguimiento, se encontró que ambos fueron significativos en el postratamiento (modelo 2a) y sucesos vitales, y escolaridad en el seguimiento (modelo 3a). Otros estudios también reportan que los casos que menos se benefician de las terapias son aquellos que están menos satisfechos con el apoyo social con el que cuentan, sobre todo del que se refiere a un confidente, a la pareja y a la familia, ${ }^{9,20} \mathrm{y}$ los que presentan más sucesos vitales antes de la intervención. ${ }^{6,7}$ No obstante, cuando también se incluyó la CES-D entre las variables predictoras (modelos $2 \mathrm{~b}$ y $3 b)$, sólo ésta fue significativa en ambos análisis, lo que indica que la influencia de los sucesos vitales y el apoyo social antes del tratamiento sobre los síntomas de depresión después del mismo, está mediada por su efecto concomitante sobre los síntomas de depresión. En este sentido, se ha visto que el nivel de síntomas de depresión antes de las intervenciones se relaciona con el nivel de dichos síntomas después de las mismas. ${ }^{46}$ En el modelo que estudió los efectos de las variables predictoras sobre la CES-D en el seguimiento se observó que la escolaridad también fue significativa y, como se observó, las de menor escolaridad se vieron más expuestas a sucesos adversos y a un menor apoyo social que, por lo visto, a lo largo del tiempo, van debilitando los efectos de la intervención.

Finalmente, la presencia de sucesos vitales y la falta de apoyo social ocurridos durante el seguimien- 
to también influyó en una menor reducción de los síntomas de depresión, de manera similar a lo que reportan otros autores. ${ }^{9}$ En cuanto al porcentaje de varianza explicada por cada uno de los modelos, se vio que aquellos que miden las variables predictoras y dependiente de manera simultánea (modelos 1 y 4) fueron los que explicaron un mayor porcentaje. Los modelos que relacionan la influencia de las variables antes del tratamiento sobre la depresión en el postratamiento y el seguimiento explican un pequeño porcentaje de la varianza.

\section{Conclusiones}

Es importante señalar que una limitación del estudio se observa en la evaluación de los sucesos vitales, la cual se realizó retrospectivamente, con lo cual se pierde confiabilidad en la medición de los datos, y otra, en que la medición del apoyo social es muy simple y no profundiza sobre el significado que tienen cada uno de los apoyos para las mujeres. Sin embargo, hay aportaciones a pesar de estas limitaciones, como son las siguientes:

Los resultados corroboran datos previos respecto de la relación entre ingreso, escolaridad y trabajo extradoméstico y los síntomas de depresión, así como entre los sucesos vitales y el apoyo social y los síntomas de depresión medidos antes de la intervención. $\mathrm{Al}$ analizar las variables en conjunto, la edad también resulta significativa, aunque con los datos con los que contamos para explicar este hecho es difícil disponer de una explicación satisfactoria. En cuanto a la influencia de los sucesos vitales y el apoyo social antes de la intervención sobre los síntomas de depresión en el postratamiento y seguimiento, aunque en una primera instancia ésta es significativa, se observa que su efecto está mediado por su alta correlación con los síntomas de depresión en la preintervención. Por otro lado, los sucesos vitales y la falta de apoyo social que ocurren en el seguimiento afectan negativamente la mejoría en los síntomas. La escolaridad es la variable sociodemográfica que muestra un efecto sobre el resultado de la intervención a los cuatro meses, en la cual se observa que las mujeres con menor escolaridad tienen menos apoyo social y experimentan más sucesos adversos, lo que limita su mejoría a más largo plazo.

Desde un punto de vista aplicado, los resultados sugieren que una manera de mejorar la efectividad de la intervención psicoeducativa para la depresión es tratar con mayor intensidad y de manera más específica a quienes presenten estos factores, ya que afectan la efectividad de la misma. Un ejemplo podría ser incluir el tema de educación de los hijos, ya que este aspecto es generador de síntomas de depresión. Otro sería diseñar una versión especial para mujeres con baja escolaridad e ingreso.

\section{Agradecimientos}

Agradecemos la colaboración de los Centros Comunitarios de Salud Mental Iztapalapa, Zacatenco y Cuauhtémoc y al Centro de Salud José Castro Villagrana, de la Secretaría de Salud, y a las psicólogas Maricarmen Acevedo y Socorro Luna.

\section{Referencias}

1. Monroe SM, D epue RA. Life stress and depression. En: Becker J, Kleinman A. Psychosocial aspects of depression. Hillsdale (N S): Lawrence Erlbaum Associates; 1991.

2. Brown GW, Harris T. Social origins of depression;A reply. Psychol Med 1978; 8: 577-588.

3. Brown GW, Harris TO, Hepworth C. Loss, humiliation and entrapment among women developing depression:A patient and nonpatient comparison. Psychol Med 1995; 25:7-21.

4. Paykel ES. Life events and affective disorders. Acta Psychiatr Scand 2003;108 (Suppl 418):61-66.

5. Jenaway A, Paykel ES. Life events and depression. En: HonigA, van Praga HM. D epression: N eurobiological and therapeutic advances. Chichester: John W iley \& Sons; 1997.

6. Ravindran A, Matheson K, G riffiths J, Merali Z, A nisman H. Stress, coping, uplifts, and quality of life in subtypes of depression:A conceptual frame and emerging data. J Affec D isord 2001; 71: 121-130.

7. Rojo-Moreno L, Livianos-Aldana L, Cervera-Martínez G, D omínguezC arabantes JA, Reig-C ebrian MJ.The role of stress in the onset of depressive disorders. A controlled study in a Spanish clinical sample. Soc Psychiatry Psychiatr Epidemiol 2001; 37:592-598.

8. Muñoz RF,YingY.The prevention of depression. Research and practice. Baltimore (MD): Hemisphere Publishing; 1993.

9. Zlotnick C, Shea MT, Pilkonis PA, Elkin I, Ryan C. Gender, type of treatment, dysfunctional attitudes, social support, life events, and depressive symptoms over naturalistic follow-up. Am J Psychiatry 1996;153(8):1021-1027.

10. Salgado de Snyder VN . El impacto del apoyo social y la autoestima sobre el estrés y la sintomatología depresiva en esposas de emigrantes a los Estados Unidos. An Inst Mex Psiquiatr 1992;3:83-98.

11. Romanov K, Varjonen J, Kaprio J, Koskenvuo M. Life events and depressiveness - the effect of adjustment for psychosocial factors, somatic health and genetic liability. Acta Psychiatr Scand 2003;107(1):2533.

12. Monroe SM, Harkness K, Simons AD, Thase, ME. Life stress and the symptoms of mayor depression.J N erv Men D is 2001;189:168-175. 13. Becker J, Schmaling K. Interpersonal aspects of depression from psychodynamic and attachment perspectives. En: Becker J, Kleinman A. Psychosocial aspects of depression. Hillsdale (N S): Lawrence Erlbaum Associates; 1991.

14. W ade T, Kendler K. Absence of interactions between social support and stressful life events in the prediction of major depression and depressive symptomatology in women. Psychol Med 2000;30:965-974. 15. Flaherty JA, G aviria FM, Black EM, Altman E, Mitchell T.The role of social support in the functioning of patients with unipolar depression. Am J Psychiatry 1983;140(4):473-476. 
16. Paykel ES.The evolution of life events research in psychiatry. J Affec Disord 2001; 62: 141-149.

17. Murray J. Prevention of anxiety and depression in vulnerable groups. Londres: G askell; 1995.

18. Pattinson EM.A theoretical-empirical base for social system therapy. Citado en: Monroe SM, D epue RA. Life stress and depression. En:

Becker J, Kleinman A, ed. Psychosocial aspects of depression. Hillsdale (N J): Lawrence Erlbaum Associates; 1991.

19. Cohen S,W ills TA. Stress, social support, and the buffering hypothesis. Psychol Bull 1985;98:310-357.

20. Ezquiaga E, García A, Bravo F, Pallarés T. Factors associated with outcomes in major depression:A 6-month prospective study. So C Psychiatry Psychiatr Epidemiol 1998;33:552-557.

21. Hirschfeld RMA, Klerman GL,Andreasen NC, Clayton PJ, Keller MB. Psycho-social predictors of chronicity in depressed patiens. $\mathrm{Br}$ J Psychiatry 1986;148:648-654.

22. Piccinelli M, Gómez-Homen F. G ender differences in the epidemiology of affective disorders and schizophrenia. Division of Mental Health and Prevention of Substance A buse. Ginebra:W orld Health 0 rganization; 1997.

23. Berenzon S, Medina-Mora ME, López EK, G onzález J. Prevalencia de trastornos mentales y variables asociadas en cuatro comunidades del sur de la ciudad de México. Rev Mex Psicol 1998; 15 (2): 177-185. 24.VegaW A, Kolody B,A guilar-G axiola S,A Iderete E, C atalano R, Caraveo-Anduaga J. Lifetime prevalence of DSM-III-R psychiatric disorders among urban and rural Mexican Americans in California. Arch Gen Psychiatry 1998:55:771-778.

25. Lara MA, Acevedo M, López EK, Fernández M. La salud emocional y tensiones asociadas a los papeles de género en las madres que trabajan y en las que no trabajan. Salud Ment 1993;16(2):13-22.

26. Lara MA,A cevedo M, Luna S, W eckmann C, Villarreal AL, Pego C. ¿ES difícil ser mujer? Una guía sobre depresión. México, DF: Editorial Pax; 1997.

27. Lara MA, N avarro C, Rubí N A, Mondragón L. 0 utcome of two levels of intervention in low-income women with depressive symptoms.Am J 0 rthopsychiatry 2003; 73(1):35-43.

28. Lara MA, N avarro C, Rubí N A, Mondragón L.Two levels of intervention in low-income women with depressive symptoms. Compliance and programme assessment. Int J Soc Psychiatry 2003;49(1):43-57.

29. Lara MA, N avarro C, N avarrete L, Mondragón L, Rubí N A. Seguimiento a dos años de una intervención psicoeducativa para mujeres con síntomas de depresión. Salud Ment 2003;26(3):27-36.

30. Lara MA, Mondragón L, Rubí N A. Un estudio de factibilidad sobre la prevención de la depresión en las mujeres. Salud Ment 1999;22(4):41-48. 31. Mrazek PJ, Haggery RJ. Reducing risks for mental disorders. Frontiers for preventive intervention research.W ashington, DC N ational A cademy Press; 1994.
32. Radloff L.The CES-D scale:A self-report depression scale for research in the general population. Appl Psychol Measurement 1977; 1: 385-401.

33. Spitzer RL,W illiams JBW, Gibbon M. Structural clinical interview for DSM-III-R, patient version (versión en español). N uevaYork (N Y): N ew York State Psychiatry Institute, Biometrics Research D epartment; 1988. 34. Medina-Mora ME,Tapia R, Mariño MC, Juárez F,Villatoro J, Caraveo J, Gómez M.Trastornos emocionales en una población urbana mexicana, resultados de un estudio nacional. Anales del Instituto Mexicano de Psiquiatría; 1992;3:48-55.

35.Valdés M, De Flores T. Psicobiología del estrés. Barcelona: Martínez Roca; 1985.

36. González-Forteza C, Morales F, Gutiérrez E. D etección oportuna de pacientes gineco-obstétricas con disfunción psicosocial. Ginecol 0 bstet Mex 1993:61:15-21.

37. Ullah P, Banks M, W arr P. Social support, social pressures and psychological distress during unemployment. Psychol Med 1985;15: 283-295.

38. Swindle RW, Cronkite RC, Moos RH. Life stressors, social resources, coping, and the 4-year course of unipolar depression. J Abnorm Psychol 1989:98(4):468-477.

39.Valdespino JL, 0 láiz G, López-Barajas MP, Mendoza L, Palma O, Velázquez 0 et al. Encuesta $\mathrm{N}$ acional de Salud 2000.Tomo I.Vivienda, población y utilización de servicios de salud. Cuernavaca, Morelos, México: Instituto N acional de Salud Pública; 2003. 40. MCG rath E, Keita G, Strickland B, Russo N. W omen and depression. Risk factors and treatment issues. Final report of the A merican Psychological Association's N ational Task Force on W omen and Depression. W ashington, DC:A mérican Psychiological Association; 1990. 41. Rojas G, González I, Díaz B, Miranda E, López F.A contecimientos vitales y patología psiquiátrica en el nivel primario de atención. Acta Psiquiátrica y Psicológica de América Latina 1997; 43 (2): 134-140. 42. Rojas G, Araya R, Fritsch R. Salud mental, problemas psicosociales y atención primaria de salud.Acta Psiquiátrica y Psicológica de A mérica Latina 2000; 46 (2): 119-126.

43. Lara MA, A cevedo M, Berenzon S. La depresión femenina vista desde la subjetividad de las mujeres. Cad Saude Publica 2004;19:109-118.

44. N atera G, C asco M, Herrejón E, Mora J. Interacción entre parejas con diferente patrón de consumo de alcohol y su relación con antecedentes familiares de consumo de México. Salud Ment 1993;18: 33-43.

45. Natera G, Tiburcio M,Villatoro J. Marital violence and its relationship to excessive drinking in Mexico. Contemp D rug Probl 1997;24:787-804 46. Tedlow J, Fava M, U ebelacker L, N ierenberg AA, A lpert JE, Rosenbaun J. 0 utcome definitions and predictors in depression. Psychother Psychosom 1998;67:266-270. 